\title{
Metastatic Squamous Cell Carcinoma of the Tonsil Mimicking Choroidal Melanoma
}

\author{
Elaine M. Binkley ${ }^{a} \quad$ Aron D. Sampson ${ }^{a}$ Nasreen A. Syed ${ }^{a, b} \quad$ H. Culver Boldt ${ }^{a}$ \\ ${ }^{a}$ Department of Ophthalmology and Visual Science, University of lowa Hospitals and Clinics, lowa City, IA, USA; \\ ${ }^{b}$ Department of Pathology, University of lowa Hospitals and Clinics, lowa City, IA, USA
}

\section{Established Facts}

- Many types of primary malignancies can metastasize to the choroid, most commonly breast and lung cancer.

- Metastatic lesions are typically less than $3 \mathrm{~mm}$, yellow-to-white colored, avascular, and medium-reflective-high reflective on standardized echography.

\section{Novel Insights}

- Squamous cell carcinoma of the tonsil can metastasize to the choroid.

- Choroidal metastasis can resemble choroidal melanoma both clinically and echographically.

- This may be related to unique features of the underlying primary tumor.

\section{Keywords}

Choroidal metastasis · Melanoma · Squamous cell carcinoma

\section{Abstract \\ We describe a case of a 53-year-old man with a history of metastatic squamous cell carcinoma of the tonsil who pre- sented with a large, dome-shaped, choroidal mass in the left eye with concern for extra-ocular extension. Standardized echography showed a choroidal mass with low-to-medium internal reflectivity, pockets of very low reflectivity, positive angle kappa, and 1+ vascularity that were concerning for pri-}

mary choroidal melanoma. Biopsy of the lesion was consistent with metastatic squamous cell carcinoma of the tonsil rather than melanoma. This is only the second reported case of metastatic squamous cell carcinoma to the choroid presenting with a large vascular tumor with echographic characteristics consistent with choroidal melanoma. The unique vascularity present in some cases of squamous cell carcinoma of the tonsil may explain the vascularity and large height of this patient's lesion. Tumor fine-needle aspiration biopsy can play an important role in patients with choroidal lesions in the setting of unusual primary tumors.

(c) 2020 S. Karger AG, Basel karger@karger.com

(C) 2020 S. Karger AG, Basel

www.karger.com/oop

Karger!
Elaine M. Binkley

Department of Ophthalmology and Visual Sciences

University of Iowa Hospitals and Clinics

200 Hawkins Drive, Iowa City IA, 52242 (USA)

elaine-binkley@uiowa.edu 


\section{Introduction}

The most common malignant choroidal tumors in adults are metastases from other sites [1]. These lesions are most often secondary to breast or lung cancer, and classically present as single or multiple yellow-to-white lesions in the posterior pole that are less than $3 \mathrm{~mm}$ in height, avascular, and have medium-to-high reflectivity on standardized echography [2]. These features are in contrast to choroidal melanomas which are often more heavily pigmented, vascular, and with low reflectivity on ultrasound [2].

A number of less common malignancies can present with metastasis to the choroid. In particular, metastases from squamous cell carcinoma of a variety of different origins have been described in the literature [3-9]. How-

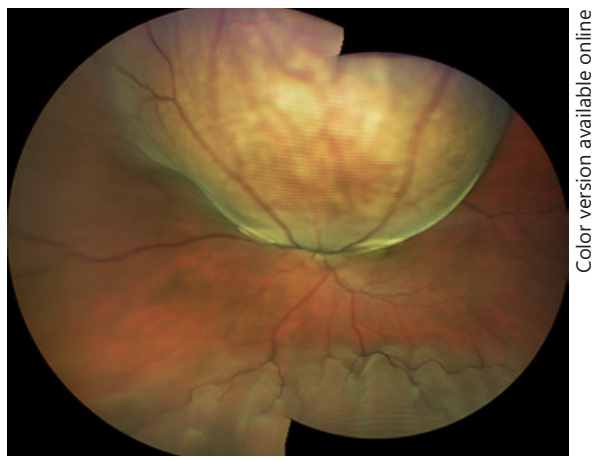

Fig. 1. Color fundus photograph of the left eye at presentation. Centered in the superior mid-periphery, there was a dome-shaped, amelanotic, vascularized choroidal mass with a lightly pigmented border. The lesion overhung the optic disc and superior macula. There was an inferior exudative retinal detachment.

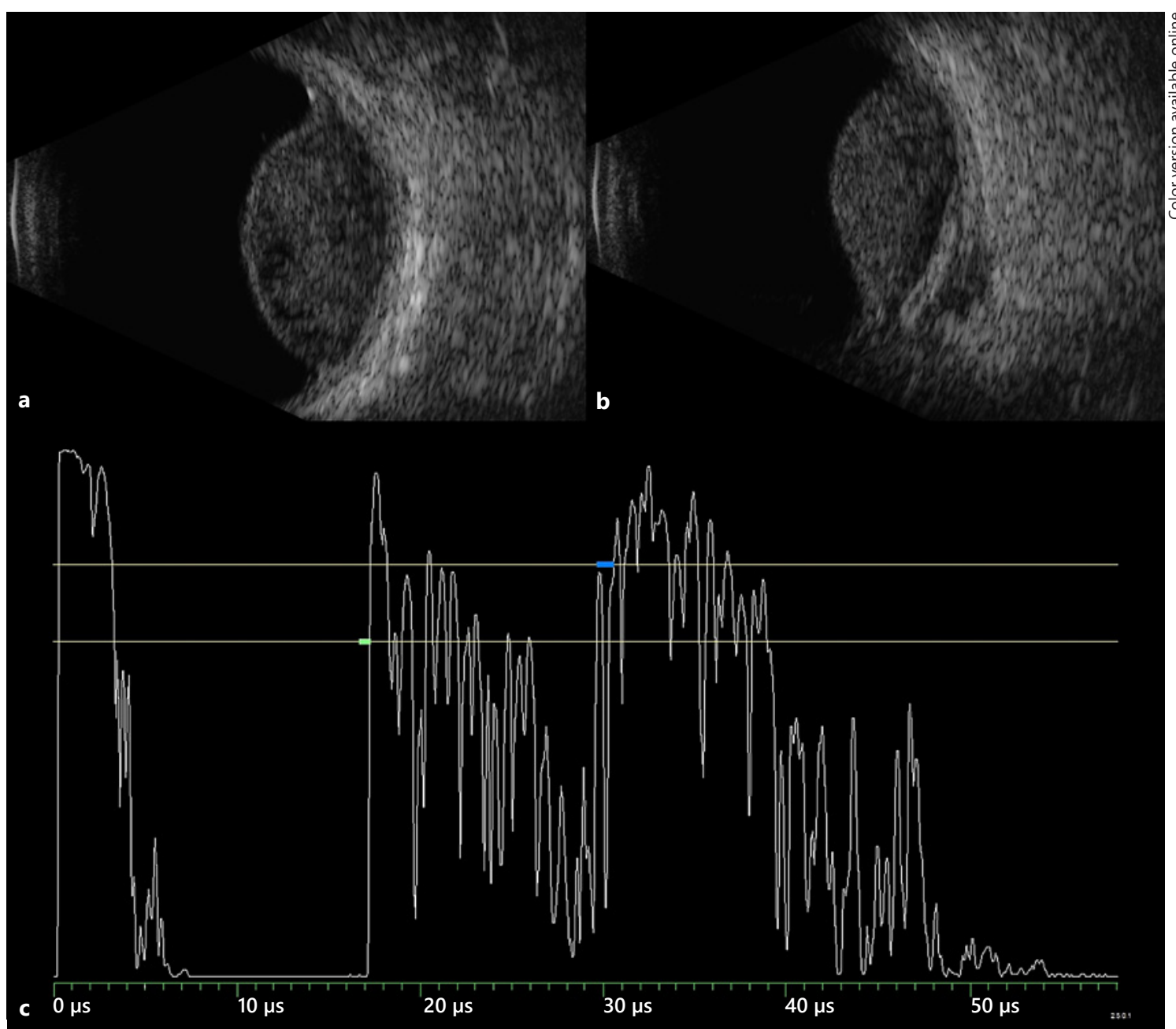

Fig. 2. Standardized echography at presentation. B-scan echography (a transverse 1130, b longitudinal 1130) and A-scan (c) revealed a dome-shaped choroidal mass measuring $18.6 \mathrm{~mm}$ in the largest basal diameter and 10.3 $\mathrm{mm}$ in height. The lesion had medium-to-low reflectivity with pockets of very low reflectivity, $1+$ vascularity, and an area concerning for extraocular extension superior to the optic nerve measuring $2.8 \mathrm{~mm}$. 
Fig. 3. Photomicrographs illustrating histology and immunohistochemistry from biopsy specimen. a There are aggregates of epithelioid cells with pleomorphic nuclei, prominent nucleoli, and abundant eosinophilic cytoplasm (hematoxylin and eosin stain; original magnification $=300 \times$ ). b Immunohistochemistry with pancytokeratin is positive in the tumor cells (immunoperoxidase; original magnification = 200x). c Tumor cell nuclei are positive for p63 consistent with squamous differentiation of the tumor (immunoperoxidase; original magnification $=200 \times)$. $\mathbf{d}$ Tumor cells are also positive for p16 immunohistochemistry, suggesting overexpression of the tumor suppressor protein due to human papillomavirus (immunoperoxidase; original magnification $=200 \times)$.

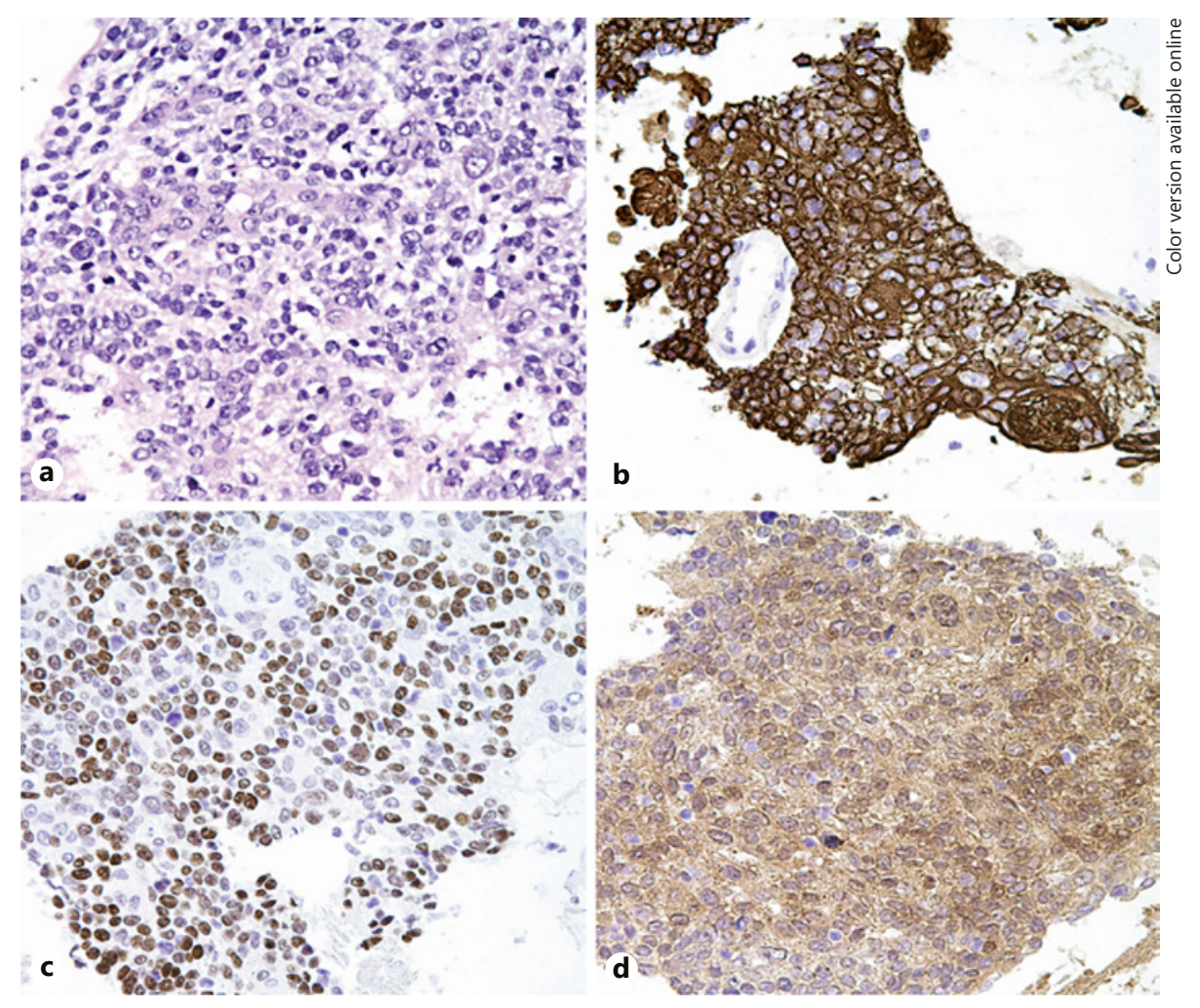

ever, most of these, with the exception of that reported by Medina, Biscotti, and Singh have had a clinical appearance typical for choroidal metastasis [9]. Here, we present a case of metastatic squamous cell carcinoma of the tonsil with features resembling large choroidal melanoma.

\section{Case Report}

A 53-year-old man with a history of HPV-positive squamous cell carcinoma of the tonsil presented for evaluation of a choroidal mass in the left eye. His disease had been staged as T3N2M0, however, he subsequently developed lung metastases that were treated with carboplatin, cetuximab, and radiation followed by nodal and pulmonary recurrence treated with carboplatin and 5-fluorouracil 9 months prior to presentation. He developed progression of disease while on maintenance cetuximab that was treated with radiation with partial response. He started pembrolizumab therapy approximately 1.5 weeks prior to presentation. He complained of a loss of the inferior half of his visual field in the left eye over a period of 3 weeks, and a retina specialist found a large, superonasal, choroidal mass with associated exudative retinal detachment. His last eye exam was 12 years prior to this episode with no history of choroidal nevus. He was referred to the Ocular Oncology service at the University of Iowa.

On examination in our clinic, visual acuity was $20 / 20-1$ in the right eye and 20/125 in the left eye. Intraocular pressure was nor- mal in each eye. Anterior segment examination was unremarkable except for age-related nuclear sclerosis in each eye. Dilated funduscopic examination in the right eye was unremarkable. In the left eye, the optic disc and superior macula were obscured by a 19.0 $\times 17.0 \times 10.3-\mathrm{mm}$ dome-shaped, amelanotic, vascular, choroidal lesion with lightly pigmented borders, and a $60 \%$ exudative retinal detachment (Fig. 1). Standardized echography of the left eye revealed a dome-shape choroidal mass with low-to-medium internal reflectivity, pockets of very low reflectivity, positive angle kappa, 1+ vascularity, and concern for extraocular extension measuring $2.8 \mathrm{~mm}$ in elevation (Fig. 2). The patient elected to undergo fine-needle aspiration biopsy of the tumor prior to definitive treatment. Pars plana vitrectomy with a 25 -gauge cutter was used to perform a biopsy of the tumor. Cytology was consistent with metastatic squamous cell carcinoma with immunophenotype suggestive of the patient's known tonsillar primary (Fig. 3). He was treated with external beam radiation with a total dose of 3,000 cGy over 10 fractions. Two weeks following radiation treatment, there was a marked reduction in tumor height to $2.4 \mathrm{~mm}$. Vision remained hand motion with a persistent inferior exudative retinal detachment for which he elected observation. Seven months following treatment, the lesion had decreased to $1.0 \mathrm{~mm}$ in thickness and the exudative detachment had resolved with return to 20/40 visual acuity (Fig. 4). He subsequently elected to transfer care locally due to the distance from our center. He developed recurrent metastasis to the liver, lungs, lymph nodes, and esophagus that have been treated with a combination of radiation and chemotherapy. He remains alive 15 months following presentation to our center. 
Fig. 4. Imaging 7 months following presentation. Color fundus photograph of the left eye (a) shows marked reduction in lesion height with residual chorioretinal scarring at the site of the mass. Standardized echography (b) shows marked reduction in lesion thickness (T1130P).

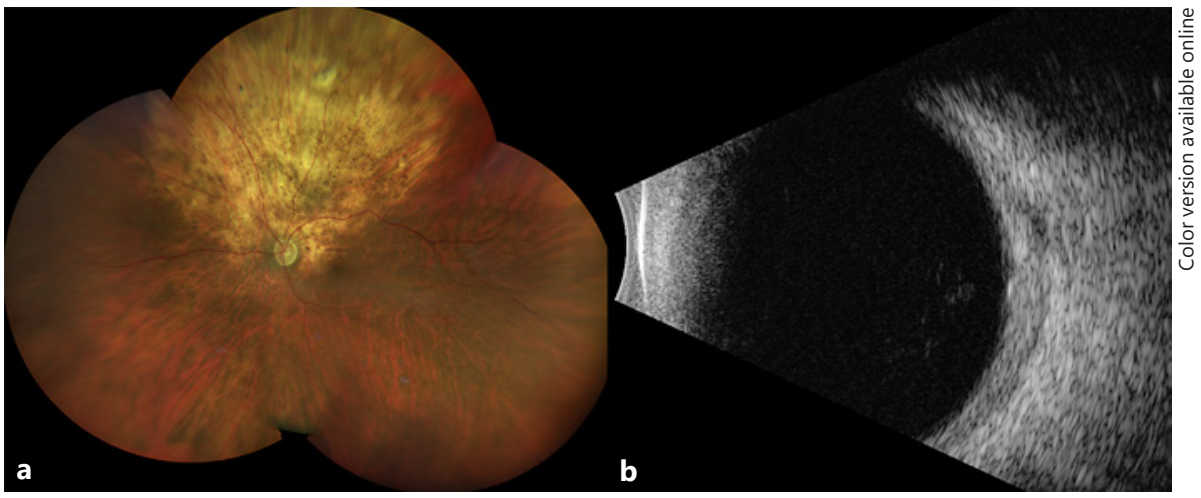

Conclusion

In conclusion, choroidal metastasis can resemble choroidal melanoma and there may be certain primary malignancies whose growth pattern overlaps choroidal melanoma. Caution should be taken prior to proceeding with primary treatment in patients with unusual primary malignancies. Tumor fine-needle aspiration biopsy plays an important role in unusual cases.

\section{Statement of Ethics}

The subject gave written informed consent for publication of this case and imaging. The IRB at the University of Iowa determined that this report does not meet the regulatory definition of human subjects research and did not require formal IRB review since it is a single case.

\section{Conflict of Interest Statement}

The authors have conflicts of interest to declare.

\section{Funding Sources}

There was no funding for this project.

\section{Author Contributions}

Each author fulfilled the ICGME criteria for authorship (acquisition and analysis E.B., A.S., N.S., C.B.), drafting and revising for intellectual content (E.B., N.S., C.B.), final approval of the version to be published (E.B., A.S., N.S., C.B.). 


\section{References}

1 Bloch RS, Gartner S. The incidence of ocular metastatic carcinoma. Arch Ophthalmol. 1971;85(6):673-75. https://doi.org/10.1001/ archopht.1971.00990050675005.

2 Agrawal A. Gass' Atlas of Macular Diseases. 5th ed. Philadelphia: Elsevier Saunders; 2012.

3 Chang SY, Tsai SH, Chen LJ, Chan WC, Tsao YP. Choroidal metastasis from esophageal squamous cell carcinoma. Taiwan J Ophthalmol. 2018 Apr-Jun;8(2):104-7.

4 Marioni G, Doro D, Marino F, Verdecchia P, Staffieri C, Staffieri A. Skin and eye: uncommon sites of distant metastasis from tongue base squamous cell carcinoma. Acta Otolaryngol. 2003 Dec;123(9):1110-4.
5 Asteriou C, Konstantinou D, Kleontas A, Paliouras D, Samanidis G, Papadopoulou F, et al. Blurred vision due to choroidal metastasis as the first manifestation of lung cancer: a case report. World J Surg Oncol. 2010 Jan;8(1):2.

6 Inoue K, Numaga J, Kaji Y, Toda J, Kato S, Sakurai M, et al. Bilateral choroidal metastases secondary to uterocervical carcinoma of the squamous cell type. Am J Ophthalmol. 2000 Nov;130(5):682-4.

7 Biswas J, Krishnakumar S, Bhavsar K, Shanmugam MP. Choroidal metastasis of a gingival squamous cell carcinoma. Am J Ophthalmol. 2002 May;133(5):713-5.
8 Samanta DB, Bhuyan R, Mishra S, Senapati S Ophthalmic metastasis in squamous cell carcinoma of head and neck: a study on two patients. Int J Sci Stud. 2015;3(2):230-3.

9 Medina C, Biscotti C, Singh AD. Squamous cell carcinoma metastatic to the choroid. J Cytol Histol. 2014;5(3):1-2.

10 Byrne S, Green R. Ultrasound of the eye and orbit. 2nd ed. New Delhi, India: Jaypee Brothers Medical Publishers; 2010. p. 119.

11 Lukes P, Pácová H, Kucera T, Veselý D, Martínek J, Astl J. Expression of endothelial and inducible nitric oxide synthase and caspase-3 in tonsillar cancer, chronic tonsillitis and healthy tonsils. Folia Biol (Praha). 2008;54(5): 141-5. 